Japan. J. Math.

Vol. 29, No. 2, 2003

\title{
Coherent sheaves on a fat curve
}

\author{
By Jin-Gen YANG \\ (Received November 16, 2001) \\ (Revised December 12, 2002) \\ (from Nagoya Mathematical Journal)
}

\begin{abstract}
The classification of coherent sheaves of pure dimension on a nonreduced smooth curve is studied.
\end{abstract}

\section{Introduction}

The classification theory of vector bundles over a nonsingular curve is welldeveloped. For reduced and irreducible singular curves there are similar theories. In this case the notion of vector bundles is replaced by torsion-free sheaves. Later it was discovered that over an arbitrary projective variety the generalization of the concept of torsion-free sheaves is that of sheaves of pure dimension ([7], [4]). It was proved in [7] that for any given Hilbert polynomial on a given projective variety the coarse moduli of semistable sheaves of pure dimension exists and is projective.

There are two directions in the study of sheaves of pure dimension. The first one is to increase the dimension of the base variety, but still restricted to the nonsingular case. For instance the problems of vector bundles over an algebraic surfaces have been extensively studied. Another direction is to consider sheaves of pure dimension over an irreducible but non-reduced curve, which we are taking in this paper.

The moduli schemes for such objects have complicated structures. Except for very special cases, the moduli schemes are neither irreducible nor reduced. Let $C$ be a projective irreducible scheme of dimension 1 over an algebraic closed field $k$. Let $\mathcal{I}$ be an ideal sheaf such that $C_{\text {red }}=\left(C, \mathcal{O}_{C} / \mathcal{I}\right)$ and $\mathcal{I}^{2}=0$. Then $\mathcal{I}$ is a sheaf on $C_{\text {red }}$. We say that $C$ is an infinitesimal extension of $C_{r e d}$ by $\mathcal{I}$. This is what a "fat" curve means in the title. Another major restriction in this paper is that $C_{\text {red }}$ is assumed to be nonsingular. Note that even in the case where $C_{r e d}=\mathbb{P}^{1}$ the results are highly non-trivial.

The materials are organized as follows. In Section 2, we discuss the notion

2000 Mathematics Subject Classification. Primary 14D20; Secondary 14H60.

Supported by the National Natural Science Foundation of China. 
of infinitesimal extension of a variety by a coherent sheaf and we show that every coherent sheaf on an infinitesimal extension of $X$ by a sheaf $\mathcal{M}$ is an extension of a torsion-free sheaf on $X$ by another. This enables us to make extensive use of technique of extensions, which was first applied by M.F. Atiyah in [1] to study the classification of vector bundles over an elliptic curve. The results in Section 2 can be applied to general projective varieties. Section 3 contains the main result of this paper. The idea can be explained as follows: Take two torsion-free sheaves $\mathcal{L}^{\prime}$ and $\mathcal{L}^{\prime \prime}$ on an irreducible and reduced curve $C$. Let $C^{\prime}$ be an infinitesimal extension of $C$ by a coherent sheaf $\mathcal{I}$. Regard both $\mathcal{L}^{\prime}$ and $\mathcal{L}^{\prime \prime}$ as sheaves on $C^{\prime}$. Then classify all pure sheaves $\mathcal{F}$ on $C^{\prime}$ which are extensions of $\mathcal{L}^{\prime \prime}$ by $\mathcal{L}^{\prime}$ such that $\mathcal{I}$ is exactly the annihilator of $\mathcal{L}^{\prime}$. Since the classification of torsion-free sheaves on a nonsingular curve is known, even trivial when $C=\mathbb{P}^{1}$, the classification problem which we are studying becomes a problem of extensions. In the remaining two sections we restrict ourselves to the rank 2 pure sheaves. In Section 4 we compute the spaces of infinitesimal deformations of our objects, which shed some light on the smoothness of some points on the moduli scheme. In Section 5 the moduli schemes of pure sheaves of rank 2 over a fat line are studied in detail. It is well-known that every torsion-free sheaf on $\mathbb{P}^{1}$ is completely decomposable ([2]). However this is not the case when $\mathbb{P}^{1}$ is thickened. The case of fat line is interesting because this is the simplest non-reduced variety such that the classification of pure sheaves is nontrivial. Using results in the previous sections we are able to obtain many properties of the moduli schemes, such as number of irreducible components, the locus of intersections of different components. We have a fairly clear picture of the moduli schemes. But we still cannot determine the exact algebraic structure (i.e. structure sheaf of the moduli scheme) except for some very simple cases. As a by-product we give the definition of the generalized Jacobian of a fat line and its compactification that makes certain sense.

\section{Infinitesimal extensions}

Let $X$ be a quasi-projective variety over an algebraically closed field $k$. Let $\mathcal{M}$ be a coherent sheaf on $X$. A scheme $X^{\prime}$ is called an infinitesimal extension of $X$ by $\mathcal{M}$ if $\mathcal{O}_{X^{\prime}}$ contains an ideal sheaf $\mathcal{I}$ with $\mathcal{I}^{2}=0$ such that $\left(X^{\prime}, \mathcal{O}_{X^{\prime}} / \mathcal{I}\right) \cong\left(X, \mathcal{O}_{X}\right)$ and $\mathcal{I} \cong \mathcal{M}$ as $\mathcal{O}_{X}$-modules (cf. [3] p. 189). Note that $\mathcal{I}$ is the radical of $\mathcal{O}_{X^{\prime}}$.

The underlying topological spaces of $X$ and $X^{\prime}$ are the same. Let $U$ be an open subset of $X$. We denote the open subscheme $\left(U,\left.\mathcal{O}_{X^{\prime}}\right|_{U}\right)$ by $U^{\prime}$.

The $\mathcal{O}_{X}$-module $\mathcal{O}_{X} \oplus \mathcal{M}$ is a sheaf of $\mathcal{O}_{X}$-algebras if we define the multiplication by

$$
\left(a_{1} \oplus s_{1}\right) \cdot\left(a_{2} \oplus s_{2}\right)=a_{1} a_{2} \oplus\left(a_{1} s_{2}+a_{2} s_{1}\right) .
$$

The topological space $X$ together with the structure sheaf $\mathcal{O}_{X} \oplus \mathcal{M}$ is the trivial infinitesimal extension of $X$ by $\mathcal{M}$. 
We first list some known results without proof.

Lemma 2.1. Let $X=\operatorname{Spec}(A)$ be a nonsingular affine variety. Let $M$ be a finitely generated $A$-module. Then any infinitesimal extension of $X$ by $\tilde{M}$ is trivial.

Lemma 2.2. Let $A$ be an integral domain and let $M$ be an $A$-module. Let $A^{\prime}$ be the trivial infinitesimal extension of $A$ by $M$, which means that $A^{\prime}=A \oplus M$ and $\left(a_{1} \oplus x_{1}\right)\left(a_{2} \oplus x_{2}\right)=a_{1} a_{2} \oplus\left(a_{1} x_{2}+a_{2} x_{1}\right)$ for all $a_{1}, a_{2} \in A$ and $x_{1}, x_{2} \in M$. Let $\operatorname{Aut}_{A}\left(A^{\prime}\right)$ be the group of (ring) automorphisms $f$ of $A^{\prime}$ such that $\left.f\right|_{M}=i d$ and $f$ induces the identity map on $A=A^{\prime} / M$. Let $f$ be a map from $A^{\prime}$ to $A^{\prime}$. Then $f \in \operatorname{Aut}_{A}\left(A^{\prime}\right)$ if and only if $f(a)=a+d(\bar{a})$ for all $a \in A^{\prime}$ with $d \in \operatorname{Der}_{A}(A, M)$. Here $\bar{a}$ denotes the canonical image of $a$ in $A$.

LEMma 2.3. Let $X$ be a nonsingular variety. Then all infinitesimal extensions of $X$ by $\mathcal{M}$ are classified by $H^{1}\left(X, \mathcal{H o m}\left(\Omega_{X}, \mathcal{M}\right)\right)$ where $\Omega_{X}$ is the sheaf of differentials on $X$. The zero element in $H^{1}\left(X, \mathcal{H} o m\left(\Omega_{X}, \mathcal{M}\right)\right)$ represents the trivial extension.

Definition 2.4. Let $d$ be a non-negative integer. A coherent sheaf $\mathcal{F}$ on $X$ is called a sheaf of pure dimension $d$ if the support of every non-zero coherent subsheaf of $\mathcal{F}$ has dimension $d$. ([7], [4])

Let $X^{\prime}$ be an infinitesimal extension of $X$ by a coherent sheaf $\mathcal{M}$. Let $\mathcal{I}$ denote the radical ideal of $\mathcal{O}_{X^{\prime}}$. It means that $\mathcal{I} \cong \mathcal{M}$. Let $\mathcal{F}$ be a coherent sheaf of pure dimension $d=\operatorname{dim}(X)$ on $X^{\prime}$. For every open subset $U$ of $X^{\prime}$, assign $\mathcal{G}(U)$ to be the set of all sections $s$ in $\mathcal{F}(U)$ such that $\mathcal{I}_{p} s_{p}=0$ for every $p \in U$, where $\mathcal{I}_{p}$ is the stalk of $\mathcal{I}$ at $p$ and $s_{p}$ is the image of $s$ in $\mathcal{F}_{p}$. Then $\mathcal{G}$ is a coherent subsheaf of $\mathcal{F}$, which is denoted by $\mathcal{F}^{0}$. Obviously, $\mathcal{F}^{0}$ has an $\mathcal{O}_{X}$-module structure.

Lemma 2.5. Let $\mathcal{F}$ be a coherent sheaf of pure dimension $d=\operatorname{dim}(X)$ on $X^{\prime}$. Then $\mathcal{F} / \mathcal{F}^{0}$ is a torsion-free $\mathcal{O}_{X}$-module.

Proof. Since $\mathcal{I F}^{0}=0$, the quotient sheaf $\mathcal{F} / \mathcal{F}^{0}$ has an $\mathcal{O}_{X}$-module structure. Let $p$ be an arbitrary point in $X^{\prime}$ and let $s \in \mathcal{F}_{p} \backslash \mathcal{F}_{p}^{0}$. Let $a \in \mathcal{O}_{X^{\prime}, p}$ such that $a s \in \mathcal{F}_{p}^{0}$. Since $s \notin \mathcal{F}_{p}^{0}$, there exists $b \in \mathcal{I}_{p}$ such that $b s \neq 0$. Since $\mathcal{F}$ is of pure dimension $d$, the annihilator of $b s$ in $\mathcal{O}_{X^{\prime}, p}$ is contained in $\mathcal{I}_{p}$. Since $a(b s)=0$, it follows that $a \in \mathcal{I}_{p}$. Hence $\mathcal{F}_{p} / \mathcal{F}_{p}^{0}$ is a torsion-free $\mathcal{O}_{X, p}$-module.

Definition 2.6. Let $\mathcal{F}$ be a pure sheaf on $X^{\prime}$. The rank of $\mathcal{F}$ is defined to be the sum of the ranks of $\mathcal{O}_{X}$ sheaves $\mathcal{F}^{0}$ and $\mathcal{F} / \mathcal{F}^{0}$, denoted by $r(\mathcal{F})$.

\section{Pure sheaves over an infinitesimal extension of a curve}

Let $C$ be a nonsingular curve over an algebraically closed field $k$. Let $C^{\prime}$ be an infinitesimal extension of $C$ by a coherent sheaf $\mathcal{M}$ on $C$. Let $\mathcal{I}$ denote the radical 
ideal of $\mathcal{O}_{C^{\prime}}$. A coherent sheaf of pure dimension 1 will simply be called a pure sheaf.

Let $\mathcal{F}$ be a pure sheaf on $C^{\prime}$. Denote $\mathcal{F}^{0}$ and $\mathcal{F} / \mathcal{F}^{0}$ by $\mathcal{E}$ and $\mathcal{E}^{\prime}$ respectively. If $\mathcal{E}^{\prime} \neq 0$ then we say that $\mathcal{F}$ is of type I, otherwise it is of type II.

According to Lemma 2.5 both $\mathcal{E}$ and $\mathcal{E}^{\prime}$ are locally free sheaves on $C$. The sequence

$$
0 \rightarrow \mathcal{E} \rightarrow \mathcal{F} \stackrel{p}{\rightarrow} \mathcal{E}^{\prime} \rightarrow 0
$$

is exact.

LeMma 3.1. Let $C^{\prime}$ be an infinitesimal extension of a nonsingular curve $C$ by a coherent sheaf $\mathcal{M}$. Let $\mathcal{L}$ be an invertible sheaf on $C$. Then there exists an invertible sheaf $\mathcal{L}^{\prime}$ on $C^{\prime}$ such that

$$
\mathcal{L}^{\prime} \otimes \mathcal{O}_{C} \cong \mathcal{L}
$$

Proof. This follows from the short exact sequence

$$
0 \rightarrow \mathcal{M} \rightarrow \mathcal{O}_{C^{\prime}}^{*} \rightarrow \mathcal{O}_{C}^{*} \rightarrow 0
$$

and the vanishing of $H^{2}(C, \mathcal{M})$.

LEMMA 3.2. Let $C^{\prime}$ be an infinitesimal extension of a nonsingular curve $C$ by a coherent sheaf $\mathcal{M}$. Let $\mathcal{L}$ be an invertible sheaf on $C$. Let $\mathcal{E}$ be a coherent sheaf on $C$. Then

$$
\mathcal{E} x t_{\mathcal{O}_{C^{\prime}}}^{1}(\mathcal{L}, \mathcal{E}) \cong \mathcal{H o m}_{\mathcal{O}_{C}}(\mathcal{M} \otimes \mathcal{L}, \mathcal{E})
$$

Proof. By Lemma 3.1 there exists an invertible sheaf $\mathcal{L}^{\prime}$ on $C^{\prime}$ such that $\mathcal{L}^{\prime} \otimes \mathcal{O}_{C} \cong \mathcal{L}$. Thus we have an exact sequence

$$
0 \rightarrow \mathcal{M} \otimes \mathcal{L} \rightarrow \mathcal{L}^{\prime} \rightarrow \mathcal{L} \rightarrow 0
$$

Since $\mathcal{E} x t_{\mathcal{O}_{C^{\prime}}}^{1}\left(\mathcal{L}^{\prime}, \mathcal{E}\right)=0$, we obtain an exact sequence

$$
\begin{aligned}
\mathcal{H o m}_{\mathcal{O}_{C^{\prime}}}(\mathcal{L}, \mathcal{E}) & \stackrel{f}{\rightarrow} \mathcal{H o m}_{\mathcal{O}_{C^{\prime}}}\left(\mathcal{L}^{\prime}, \mathcal{E}\right) \rightarrow \mathcal{H o m} \text { O }_{C}(\mathcal{M} \otimes \mathcal{L}, \mathcal{E}) \\
& \rightarrow \mathcal{E} x t_{\mathcal{O}_{C^{\prime}}}^{1}(\mathcal{L}, \mathcal{E}) \rightarrow 0
\end{aligned}
$$

It is easy to see that $f$ is an isomorphism.

Let $\mathcal{E}$ and $\mathcal{G}$ be coherent sheaves on $C$. If they are regarded as $\mathcal{O}_{C^{\prime}}$-modules, it is known that the isomorphism classes of extensions of $\mathcal{G}$ by $\mathcal{E}$ are classified by $\operatorname{Ext}_{\mathcal{O}_{C^{\prime}}}^{1}(\mathcal{G}, \mathcal{E})$. 
Lemma 3.3. Let $C^{\prime}$ be an infinitesimal extension of a nonsingular curve $C$ by a coherent sheaf $\mathcal{M}$. Let $\mathcal{L}$ be an invertible sheaf on $C$. Let $\mathcal{E}$ be a coherent sheaf on $C$. Then

$$
\operatorname{Ext}_{\mathcal{O}_{C^{\prime}}}^{1}(\mathcal{L}, \mathcal{E}) \cong H^{1}\left(C, \mathcal{L}^{-1} \otimes \mathcal{E}\right) \oplus H^{0}\left(C, \mathcal{H} \operatorname{mom}_{\mathcal{O}_{C}}(\mathcal{M} \otimes \mathcal{L}, \mathcal{E})\right)
$$

Proof. Since $\operatorname{dim}(C)=1$, the second cohomology of every coherent sheaf on $C^{\prime}$ vanishes. Thus the Leray spectral sequence takes the form

$$
0 \rightarrow H^{1}\left(\mathcal{H} o m_{\mathcal{O}_{C^{\prime}}}(\mathcal{L}, \mathcal{E})\right) \rightarrow \operatorname{Ext}_{\mathcal{O}_{C^{\prime}}}^{1}(\mathcal{L}, \mathcal{E}) \rightarrow H^{0}\left(C, \mathcal{E} t_{\mathcal{O}_{C^{\prime}}}^{1}(\mathcal{L}, \mathcal{E})\right) \rightarrow 0
$$

Applying Lemma 3.2 we obtain the desired result.

Let $\mathcal{L}$ be a fixed invertible sheaf and let $\mathcal{E}$ be a fixed torsion-free sheaf on $C$. Let $M(\mathcal{L}, \mathcal{E})$ denote the set of all isomorphism classes of coherent sheaves $\mathcal{F}$ on $C^{\prime}$ such that $\mathcal{F}^{0} \cong \mathcal{E}$ and $\mathcal{F} / \mathcal{F}^{0} \cong \mathcal{L}$. Then Lemma 3.3 suggests that $M(\mathcal{L}, \mathcal{E})$ is a subset of $\mathbb{P}\left(H^{1}\left(C, \mathcal{L}^{-1} \otimes \mathcal{E}\right) \oplus H^{0}\left(C, \mathcal{H}\right.\right.$ om $\left.\left._{\mathcal{O}_{C}}(\mathcal{M} \otimes \mathcal{L}, \mathcal{E})\right)\right)$. In the remaining part of this section we will determine this subset. For that purpose we need to work out the result of Lemma 3.3 more explicitly.

For a coherent sheaf $\mathcal{F}$ on $C^{\prime}$ let us denote the isomorphism class of $\mathcal{F}$ by $[\mathcal{F}]$.

REMARK 3.4. Let $\mathcal{L}^{\prime}$ be an invertible sheaf on $C^{\prime}$ such that $\mathcal{L}^{\prime} \otimes \mathcal{O}_{C} \cong \mathcal{L}$. Then tensoring $\mathcal{L}^{\prime-1}$ gives a 1-1 correspondence between $M(\mathcal{L}, \mathcal{E})$ and $M\left(\mathcal{O}_{C}, \mathcal{E} \otimes\right.$ $\mathcal{L}^{-1}$ ). Hence it suffices to study the case $\mathcal{L} \cong \mathcal{O}_{C}$.

Let $f \in H^{1}(C, \mathcal{E})$ and $s \in \operatorname{Hom}_{C}(\mathcal{M}, \mathcal{E})$. Assume that $s \neq 0$. We may construct an element in $M\left(\mathcal{O}_{C}, \mathcal{E}\right)$ as follows.

Take an affine open covering $C=\bigcup_{i=1}^{n} U_{i}$ with $U_{i} \cong \operatorname{Spec}\left(A_{i}\right)$. Then $\left.\mathcal{M}\right|_{U_{i}} \cong$ $M_{i}$ for some $A_{i}$-module $M_{i}$. Let $g_{i}: \mathcal{O}_{C^{\prime}}\left(U_{i}\right) \rightarrow A_{i} \oplus M_{i}$ be a trivialization map. Let $\gamma_{i j}=\left.\left.g_{j}\right|_{U_{i j}} g_{i}\right|_{U_{i j}} ^{-1}$ be the transition function of $\mathcal{O}_{C^{\prime}}$ on $U_{i j}$. Recall that the multiplication in $A_{i} \oplus M_{i}$ is given by $\left(a_{1} \oplus x_{1}\right)\left(a_{2} \oplus x_{2}\right)=a_{1} a_{2} \oplus\left(a_{1} x_{2}+a_{2} x_{1}\right)$ and $\gamma_{i j}(a \oplus x)=a \oplus\left(d_{i j}(a)+\psi_{i j}(x)\right)$ where $\psi_{i j}$ is the transition function of $\mathcal{M}$ and $d_{i j}$ is a $k$-derivation from $\mathcal{O}_{C}\left(U_{i j}\right)$ into $\mathcal{M}\left(U_{i j}\right)$ such that

$$
d_{i l}=d_{j l}+\psi_{j l} d_{i j}
$$

for any $1 \leq i<j<l \leq n$.

The element $f \in H^{1}(C, \mathcal{E})$ is represented by a 1-cocycle $\left\{f_{i j}\right\}_{1 \leq i<j \leq n}$ of $\mathcal{E}$. That is to say, $f_{i j} \in \mathcal{E}\left(U_{i j}\right)$ and

$$
f_{i l}=f_{j l}+\mu_{j l}\left(f_{i j}\right)
$$

where $\mu_{j l}$ is the transition function of $\mathcal{E}$ from $U_{j}$ to $U_{l}$. 
The element $s \in \operatorname{Hom}_{C}(\mathcal{M}, \mathcal{E})$ satisfies the condition

$$
\mu_{i j} s=s \psi_{i j}
$$

Denote $E_{i}=\mathcal{E}\left(U_{i}\right)$. Let $N_{i}=A_{i} \oplus E_{i}$ (as an abelian group). Then $N_{i}$ has an $A_{i} \oplus M_{i}$-module structure under

$$
\left(a_{i} \oplus b_{i}\right)\left(x_{i} \oplus y_{i}\right)=a_{i} x_{i} \oplus\left(a_{i} y_{i}+x_{i} s\left(b_{i}\right)\right) .
$$

Let $\mathcal{F}_{i}=\tilde{N}_{i}$. We can glue all $\mathcal{F}_{i}$ together by the following morphisms

$$
\begin{gathered}
\phi_{i j}:\left.\left.\mathcal{F}_{i}\right|_{U_{i j}} \rightarrow \mathcal{F}_{j}\right|_{U_{i j}} \\
x_{i} \oplus y_{i} \mapsto x_{i} \oplus\left(\mu_{i j}\left(y_{i}\right)+x_{i} f_{i j}+s\left(d_{i j}\left(x_{i}\right)\right)\right) .
\end{gathered}
$$

To verify this we need to check the following two equalities:

1)

$$
\phi_{i j}\left(\left(a_{i} \oplus b_{i}\right)\left(x_{i} \oplus y_{i}\right)\right)=\gamma_{i j}\left(a_{i} \oplus b_{i}\right) \phi_{i j}\left(x_{i} \oplus y_{i}\right)
$$

2)

$$
\phi_{i l}=\phi_{j l} \phi_{i j}
$$

The left hand side of (5) is equal to

$$
\begin{aligned}
& \phi_{i j}\left(a_{i} x_{i} \oplus\left(a_{i} y_{i}+x_{i} s\left(b_{i}\right)\right)\right) \\
& \quad=a_{i} x_{i} \oplus\left(a_{i} \mu_{i j}\left(y_{i}\right)+x_{i} \mu_{i j}\left(s\left(b_{i}\right)\right)+a_{i} x_{i} f_{i j}+s\left(d_{i j}\left(a_{i} x_{i}\right)\right)\right) .
\end{aligned}
$$

The right hand side of (5) is equal to

$$
\begin{aligned}
& \left(a_{i} \oplus\left(d_{i j}\left(a_{i}\right)+\psi_{i j}\left(b_{i}\right)\right)\right)\left(x_{i} \oplus\left(\mu_{i j}\left(y_{i}\right)+x_{i} f_{i j}+s\left(d_{i j}\left(x_{i}\right)\right)\right)\right) \\
& \quad=a_{i} x_{i} \oplus\left(a_{i} \mu_{i j}\left(y_{i}\right)+a_{i} x_{i} f_{i j}+a_{i} s\left(d_{i j}\left(x_{i}\right)\right)+x_{i} s\left(d_{i j}\left(a_{i}\right)+\psi_{i j}\left(b_{i}\right)\right)\right) .
\end{aligned}
$$

If follows from (4) that (5) is valid.

Let $x_{i} \oplus y_{i}$ be an arbitrary element in $N_{i}=A_{i} \oplus E_{i}$. Then

$$
\begin{aligned}
& \phi_{j l}\left(\phi_{i j}\left(x_{i} \oplus y_{i}\right)\right) \\
& =\phi_{j l}\left(x_{i} \oplus\left(\mu_{i j}\left(y_{i}\right)+x_{i} f_{i j}+s\left(d_{i j}\left(x_{i}\right)\right)\right)\right) \\
& =x_{i} \oplus\left(\mu_{j l}\left(\mu_{i j}\left(y_{i}\right)+x_{i} f_{i j}+s\left(d_{i j}\left(x_{i}\right)\right)\right)+x_{i} f_{j l}+s\left(d_{j l}\left(x_{i}\right)\right)\right) \\
& =x_{i} \oplus\left(\mu_{i l}\left(y_{i}\right)+x_{i}\left(\mu_{j l}\left(f_{i j}\right)+f_{j l}\right)+\mu_{j l}\left(s\left(d_{i j}\left(x_{i}\right)\right)\right)+s\left(d_{j l}\left(x_{i}\right)\right)\right) .
\end{aligned}
$$

If follows from (3), (4) and (2) that

$$
\phi_{j l}\left(\phi_{i j}\left(x_{i} \oplus y_{i}\right)\right)=\phi_{i l}\left(x_{i} \oplus y_{i}\right) .
$$


Let $\mathcal{F}$ be the coherent sheaf on $C^{\prime}$ obtained by glueing $\mathcal{F}_{i}$ and $\mathcal{F}_{j}$ through $\phi_{i j}$ for all $i, j$.

The projections $\pi_{i}\left(x_{i} \oplus y_{i}\right)=x_{i}$ from $N_{i}$ to $A_{i}$ give a morphism $\pi: \mathcal{F} \rightarrow \mathcal{O}_{C}$. There is a short exact sequence

$$
0 \rightarrow \mathcal{E} \rightarrow \mathcal{F} \stackrel{\pi}{\rightarrow} \mathcal{O}_{C} \rightarrow 0
$$

Since $s \neq 0$ and $\mathcal{E}$ is torsion-free, we have $\mathcal{F}^{0}=\mathcal{E}$. Therefore $\mathcal{F}$ represents an element in $M\left(\mathcal{O}_{C}, \mathcal{E}\right)$.

Let $\left\{f_{i j}^{\prime}\right\}$ be another 1-cocycle representing $f$. Then $f_{i j}^{\prime}=f_{i j}+\rho_{U_{i} U_{i j}}\left(g_{i}\right)-$ $\rho_{U_{j} U_{i j}}\left(g_{j}\right)$ where $g_{i} \in \mathcal{E}\left(U_{i}\right)$. Let $\mathcal{F}^{\prime}$ be an element of $M\left(\mathcal{O}_{C}, \mathcal{E}\right)$ constructed from $\left\{f_{i j}^{\prime}\right\}$ and $s$. This means that $\mathcal{F}_{i}=\tilde{N}_{i}$ are glued together by the morphisms

$$
\begin{gathered}
\phi_{i j}^{\prime}:\left.\left.\mathcal{F}_{i}\right|_{U_{i j}} \rightarrow \mathcal{F}_{j}\right|_{U_{i j}} \\
x_{i} \oplus y_{i} \mapsto x_{i} \oplus\left(\mu_{i j}\left(y_{i}\right)+x_{i} f_{i j}^{\prime}+s\left(d_{i j}\left(x_{i}\right)\right)\right) .
\end{gathered}
$$

Let $\tau_{i}: N_{i} \rightarrow N_{i}, x_{i} \oplus y_{i} \mapsto x_{i} \oplus\left(y_{i}+x_{i} g_{i}\right)$. Then $\tau_{i}$ is an automorphism of the $A_{i} \oplus M_{i}$-module $N_{i}$. One can easily verify that $\tau_{j} \phi_{i j}^{\prime}=\phi_{i j} \tau_{i}$ over $U_{i j}$. Hence $\left\{\tau_{i}\right\}$ is an isomorphism from $\mathcal{F}^{\prime}$ to $\mathcal{F}$. This shows that the sheaf $\mathcal{F}$ we obtained does not depend upon the choice of representative in $H^{1}(C, \mathcal{E})$.

Let $\operatorname{Hom}_{C}(\mathcal{M}, \mathcal{E})^{*}$ denote the set of non-zero elements of $\operatorname{Hom}_{C}(\mathcal{M}, \mathcal{E})$. The above construction gives rise to a map

$$
\Phi_{0}: H^{1}(C, \mathcal{E}) \times \operatorname{Hom}_{C}(\mathcal{M}, \mathcal{E})^{*} \rightarrow M\left(\mathcal{O}_{C}, \mathcal{E}\right) .
$$

Let $\mathbb{P}\left(H^{1}(C, \mathcal{E}) \times \operatorname{Hom}_{C}(\mathcal{M}, \mathcal{E})^{*}\right)$ denote the set of all elements $[(f, s)]$ in $\mathbb{P}\left(H^{1}(C, \mathcal{E}) \times \operatorname{Hom}_{C}(\mathcal{M}, \mathcal{E})\right)$ such that $s \neq 0$.

Let $f \in H^{1}(C, \mathcal{E})$ and $s \in \operatorname{Hom}_{C}(\mathcal{M}, \mathcal{E})^{*}$. Let $c$ be a non-zero constant. It is easy to verify that $\Phi_{0}(f, s)=\Phi_{0}(c f, c s)$. Thus $\Phi_{0}$ induces a map

$$
\Phi: \mathbb{P}\left(H^{1}(C, \mathcal{E}) \times \operatorname{Hom}_{C}(\mathcal{M}, \mathcal{E})^{*}\right) \rightarrow M\left(\mathcal{O}_{C}, \mathcal{E}\right) .
$$

We claim that $\Phi$ is a bijection.

Let $\mathcal{F}$ be a pure sheaf on $C^{\prime}$ such that $\mathcal{F}^{0} \cong \mathcal{E}$ and $\mathcal{F} / \mathcal{F}^{0} \cong \mathcal{O}_{C}$. Choose isomorphisms $p: \mathcal{F}^{0} \rightarrow \mathcal{E}$ and $q: \mathcal{F} / \mathcal{F}^{0} \cong \mathcal{O}_{C}$. The multiplication induces a homomorphism from $\mathcal{M} \otimes \mathcal{F}$ into $\mathcal{F}^{0}$. Since $\mathcal{M}$ annihilates $\mathcal{F}^{0}$, This is actually a homomorphism from $\mathcal{M} \otimes\left(\mathcal{F} / \mathcal{F}^{0}\right)$ into $\mathcal{F}^{0}$. Hence we obtain an element $s \in$ $\operatorname{Hom}_{C}(\mathcal{M}, \mathcal{E})$. By the definition of $\mathcal{F}^{0}$ the element $s$ is not zero.

Let $\partial: H^{0}\left(C, \mathcal{O}_{C}\right) \rightarrow H^{1}(C, \mathcal{E})$ be the connection morphism of the short exact sequence

$$
0 \rightarrow \mathcal{E} \rightarrow \mathcal{F} \rightarrow \mathcal{O}_{C} \rightarrow 0
$$


Let $f=\partial(1)$. Note that $f$ and $s$ depend upon the choices of isomorphisms $p$ and $q$. For a different choice of isomorphisms $p^{\prime}$ and $q^{\prime}$, assume that we obtain $f^{\prime} \in H^{1}(C, \mathcal{E})$ and $s^{\prime} \in \operatorname{Hom}_{C}(\mathcal{M}, \mathcal{E})$. Then it is easy to see that there is a nonzero constant $c$ such that $f^{\prime}=c f$ and $s^{\prime}=c s$. Thus $[\mathcal{F}]$ determines an element $[(f, s)] \in \mathbb{P}\left(H^{1}(C, \mathcal{E}) \times \operatorname{Hom}_{C}(\mathcal{M}, \mathcal{E})^{*}\right)$. Therefore we obtain a map

$$
\Psi: M\left(\mathcal{O}_{C}, \mathcal{E}\right) \rightarrow \mathbb{P}\left(H^{1}(C, \mathcal{E}) \times \operatorname{Hom}_{C}(\mathcal{M}, \mathcal{E})^{*}\right) .
$$

It is a matter of routine to check that $\Phi \Psi=i d$ and $\Psi \Phi=i d$. Using Remark 3.4 we obtain the following results.

THEOREM 3.5. Let $C$ be a complete nonsingular curve over an algebraically closed field $k$. Let $\mathcal{L}$ be an invertible sheaf on $C$. Let $\mathcal{M}$ be a coherent sheaf on $C$ and let $\mathcal{E}$ be a locally free sheaf on $C$. Let $C^{\prime}$ be an infinitesimal extension of $C$ by $\mathcal{M}$. The pure sheaves $\mathcal{F}$ on $C^{\prime}$ such that $\mathcal{F}^{0} \cong \mathcal{E}$ and $\mathcal{F} / \mathcal{F}^{0} \cong \mathcal{L}$ are classified by $\mathbb{P}\left(H^{1}\left(C, \mathcal{L}^{-1} \otimes \mathcal{E}\right) \times \operatorname{Hom}_{C}(\mathcal{L} \otimes \mathcal{M}, \mathcal{E})^{*}\right)$.

Next we study the families of pure sheaves in $M\left(\mathcal{O}_{C}, \mathcal{E}\right)$, where $\mathcal{E}$ is an invertible sheaf on $C$. Let $S$ be an irreducible and reduced variety. Let $X=C \times S$ and $X^{\prime}=C^{\prime} \times S$. Let $\pi_{1}$ and $\pi_{2}$ denote the projections from $X$ (or $X^{\prime}$ ) to $C$ (or $C^{\prime}$ ) and $S$ respectively.

Let $\mathcal{F}$ be a coherent sheave on $X$ flat over $S$ and $\left[\mathcal{F}_{s}\right] \in M\left(\mathcal{O}_{C}, \mathcal{E}\right)$ for every closed point $s \in S$. Then we call $\mathcal{F}$ a family of sheaves in $M\left(\mathcal{O}_{C}, \mathcal{E}\right)$. For a family $\mathcal{F}$ there is a well-defined map $\lambda: S \rightarrow \mathbb{P}\left(H^{1}(C, \mathcal{E}) \times \operatorname{Hom}(\mathcal{M}, \mathcal{E})^{*}\right)$ such that $\lambda(s)=\Psi\left(\mathcal{F}_{s}\right)$. We want to show that $\lambda$ is a morphism.

Let $\mathcal{I}$ be the sheaf of radicals of $\mathcal{O}_{X}$. Then $\mathcal{I} \cong \pi_{1}^{*}(\mathcal{M})$. Let $\mathcal{F}^{0}$ be the sheaf of sections of $\mathcal{F}$ annihilated by $\mathcal{I}$.

LeMma 3.6. Let $C$ be a nonsingular curve and let $S$ be a reduced and irreducible variety. Let $\mathcal{Q}$ be an invertible sheaf on $C$ and let $\mathcal{G}$ be a coherent sheaf on $C \times S$ such that $\mathcal{G}_{s} \cong \mathcal{Q}$ for every closed point $s \in S$. Then $\mathcal{G} \cong \pi_{1}^{*}(\mathcal{Q}) \otimes \pi_{2}^{*}(\mathcal{L})$ for some invertible sheaf $\mathcal{L}$ on $S$.

Proof. Embed $C$ in some projective space $\mathbb{P}^{N}$. Then $C \times S$ is a closed subvariety of $\mathbb{P}_{S}^{N}$. Since $\mathcal{G}_{s} \cong \mathcal{Q}$ for every closed point $s \in S$, the Hilbert polynomial of $\mathcal{G}_{s}$ does not change when $s$ changes. By [3] pp. 261-262 $\mathcal{G}$ is flat over $S$. Hence $\mathcal{G}$ is locally free by Lemma 5.4 of [5]. Let $\mathcal{G}^{\prime}=\mathcal{G} \otimes \pi_{1}^{*}\left(\mathcal{Q}^{-1}\right)$. Then $\mathcal{G}^{\prime}$ is an invertible sheaf on $C \times S$ whose restriction to each closed fibre of $\pi_{2}$ is the structure sheaf of $C$. Hence $\mathcal{G}^{\prime}$ is isomorphic to $\pi_{2}^{*}(\mathcal{L})$ for some invertible sheaf $\mathcal{L}$ on $S$.

Now return to the family $\mathcal{F}$. Since $\mathcal{F}_{s}^{0} \cong \mathcal{E}$ for every closed point $s \in S$, Lemma 3.6 implies that there is an affine open covering $S=\cup_{i} W_{i}$ of $S$ such that $\left.\left.\mathcal{F}^{0}\right|_{\pi_{2}^{-1}\left(W_{i}\right)} \cong \pi_{1}^{*}(\mathcal{E})\right|_{\pi_{2}^{-1}\left(W_{i}\right)}$ and $\mathcal{F} /\left.\mathcal{F}^{0}\right|_{\pi_{2}^{-1}\left(W_{i}\right)} \cong \mathcal{O}_{\pi_{2}^{-1}\left(W_{i}\right)}$ for each $i$. Denote the induced open subschemes of $X$ and $X^{\prime}$ on $\pi_{2}^{-1}\left(W_{i}\right)$ by $X_{i}$ and $X_{i}^{\prime}$ respectively. 
Let $g:\left.\left.\mathcal{F}^{0}\right|_{X_{i}} \rightarrow \pi_{1}^{*}(\mathcal{E})\right|_{X_{i}}$ and $h: \mathcal{F} /\left.\mathcal{F}^{0}\right|_{X_{i}} \cong \mathcal{O}_{X_{i}}$ be isomorphisms. With this choice of isomorphisms we obtain an exact sequence

$$
\left.\left.0 \rightarrow \pi_{1}^{*}(\mathcal{E})\right|_{X_{i}} \rightarrow \mathcal{F}\right|_{X_{i}^{\prime}} \rightarrow \mathcal{O}_{X_{i}} \rightarrow 0
$$

The multiplication of $\left.\mathcal{I}\right|_{X_{i}}$ with $\left.\mathcal{F}\right|_{X_{i}^{\prime}}$ determines an element

$$
t \in \operatorname{Hom}_{\mathcal{O}_{X_{i}}}\left(\left.\pi_{1}^{*}(\mathcal{M})\right|_{X_{i}},\left.\pi_{1}^{*}(\mathcal{E})\right|_{X_{i}}\right) .
$$

Let $\mathcal{B}=\pi_{2 *}\left(\mathcal{H} \operatorname{om}_{\mathcal{O}_{X_{i}}}\left(\left.\pi_{1}^{*}(\mathcal{M})\right|_{X_{i}},\left.\pi_{1}^{*}(\mathcal{E})\right|_{X_{i}}\right)\right)$. Then $t \in \Gamma\left(S_{i}, \mathcal{B}\right)$. The section $t$ defines a morphism $\mu_{i}: S_{i} \rightarrow \operatorname{Hom}_{C}(\mathcal{M}, \mathcal{E})^{*}$. Here $\operatorname{Hom}_{C}(\mathcal{M}, \mathcal{E})^{*}$ is supplied with the natural structure of quasi-affine variety.

Applying the functor $\pi_{2 *}$ to the exact sequence (7) we obtain the long exact sequence

$$
0 \rightarrow \pi_{2 *}\left(\left.\pi_{1}^{*}(\mathcal{E})\right|_{X_{i}}\right) \rightarrow \pi_{2 *}\left(\left.\mathcal{F}\right|_{X_{i}^{\prime}}\right) \rightarrow \pi_{2 *} \mathcal{O}_{X_{i}} \stackrel{\xi}{\rightarrow} R^{1} \pi_{2 *}\left(\left.\pi_{1}^{*}(\mathcal{E})\right|_{X_{i}}\right) \rightarrow \cdots
$$

Let $u=\xi(1)$. Then $u$ defines a morphism $\nu_{i}$ from $S_{i}$ into the affine variety $H^{1}(C, \mathcal{E})$. It is not hard to verify that $\lambda(s)=\left[\left(\nu_{i}(s), \mu_{i}(s)\right)\right]$ for every closed point $s \in S_{i}$. Thus $\left.\lambda\right|_{S_{i}}$ is a morphism. Therefore $\lambda$ is a morphism. The above discussion can be summarized as the following result.

Proposition 3.7. Let $\mathcal{F}$ be a family of sheaves in $M\left(\mathcal{O}_{C}, \mathcal{E}\right)$ over a reduced and irreducible variety $S$. Let $\lambda: S \rightarrow \mathbb{P}\left(H^{1}(C, \mathcal{E}) \times \operatorname{Hom}(\mathcal{M}, \mathcal{E})^{*}\right)$ be the map associated with the family $\mathcal{F}$. Then $\lambda$ is a morphism.

\section{Infinitesimal deformations of pure sheaves of rank 2}

Let $C$ be a complete nonsingular curve. Let $\mathcal{M}$ be an invertible sheaf on $C$. Let $C^{\prime}$ be an infinitesimal extension of $C$ by $\mathcal{M}$. Let $\mathcal{L}^{\prime}$ and $\mathcal{L}^{\prime \prime}$ be invertible sheaves on $C$. Let $\mathcal{F}$ be a pure sheaf on $C^{\prime}$ with $\mathcal{F}^{0} \cong \mathcal{L}^{\prime}$ and $\mathcal{F} / \mathcal{F}^{0} \cong \mathcal{L}^{\prime \prime}$. In this section we study the infinitesimal deformations of the sheaf $\mathcal{F}$.

It is known that $\operatorname{Ext}_{C^{\prime}}^{1}(\mathcal{F}, \mathcal{F})$ classifies the first order deformations of $\mathcal{F}$ and obstructions lie in $\operatorname{Ext}_{C^{\prime}}^{i}(\mathcal{F}, \mathcal{F})$ for $i>1$.

Assume that $\mathcal{F}=\Phi(f, s)$, where $f \in H^{1}\left(C, \mathcal{L}^{\prime \prime-1} \otimes \mathcal{L}^{\prime}\right)$ and $s$ is a non-zero element in $\operatorname{Hom}_{C}\left(\mathcal{L}^{\prime \prime} \otimes \mathcal{M}, \mathcal{L}^{\prime}\right)$. Then $s$ can be identified with a non-zero element in $H^{0}\left(C, \mathcal{L}^{\prime \prime-1} \otimes \mathcal{M}^{-1} \otimes \mathcal{L}^{\prime}\right)$. Note that it is necessary that $\operatorname{deg}\left(\mathcal{L}^{\prime}\right) \geq \operatorname{deg}(\mathcal{M})+$ $\operatorname{deg}\left(\mathcal{L}^{\prime \prime}\right)$. Let $D=\sum_{i=1}^{n} d_{i} z_{i}$ be the zero divisor of $s$, in which $z_{1}, \ldots, z_{n}$ are distinct points on $C$ and $d_{1}, \ldots, d_{n}$ are positive integers.

Lemma 4.1. Let $z$ be a closed point of $C$.

1) If $z \notin\left\{z_{1}, \ldots, z_{n}\right\}$, then

$$
\mathcal{E} x t_{\mathcal{O}_{C^{\prime}, z}}^{i}\left(\mathcal{F}_{z}, \mathcal{F}_{z}\right)=0
$$


for all $i>0$.

2)

$$
\mathcal{E} x t_{\mathcal{O}_{C^{\prime}, z_{j}}}^{i}\left(\mathcal{F}_{z_{j}}, \mathcal{F}_{z_{j}}\right) \cong k^{2 d_{j}}
$$

for $i>0$ and $1 \leq j \leq n$.

Proof. Let $A=\mathcal{O}_{C, z}$. Then $\mathcal{O}_{C^{\prime}, z} \cong A \oplus A$ with the multiplication $\left(a_{1} \oplus\right.$ $\left.b_{1}\right)\left(a_{2} \oplus b_{2}\right)=a_{1} a_{2} \oplus\left(a_{1} b_{2}+a_{2} b_{1}\right)$.

The stalk $\mathcal{F}_{z}$ is also isomorphic to $A \oplus A$ as an abelian group and the $A$-module structure is given by the multiplication

$$
(a \oplus b)(x \oplus y)=a x \oplus\left(a y+\phi\left(s_{z}\right) b x\right),
$$

where $\phi$ is a suitable isomorphism from $\left(\mathcal{L}^{\prime \prime-1} \otimes \mathcal{M}^{-1} \otimes \mathcal{L}^{\prime}\right)_{z}$ to $A$.

Let $f: \mathcal{O}_{C^{\prime}, z} \rightarrow \mathcal{F}_{z}$ be a map given by $f(a \oplus b)=a \oplus \phi\left(s_{z}\right) b$. Then $f$ is an $\mathcal{O}_{C^{\prime}, z}$ homomorphism. Since $s \neq 0$, the homomorphism $f$ is injective.

If $z$ is away from $D$, then $s$ does not vanish at $z$ and $\phi\left(s_{z}\right)$ is an invertible element in $A$. In this case $f$ is an isomorphism. Hence

$$
\mathcal{E}_{x t_{\mathcal{O}^{\prime}, z}}^{i}\left(\mathcal{F}_{z}, \mathcal{F}_{z}\right)=0
$$

for $i>0$.

The second statement follows from the following sublemma.

Sublemma 4.2. Let $A$ be a discrete valuation ring with $\mathbf{m}$ as its maximal ideal. Let $t \in \mathbf{m}$ be a local parameter of $A$. Let $k=A / \mathbf{m}$ be the residue field. Let $d$ be a positive integer. Let $R=A \oplus A$ be the ring with

$$
\left(a_{1} \oplus b_{1}\right)+\left(a_{2} \oplus b_{2}\right)=\left(a_{1}+a_{2}\right) \oplus\left(b_{1}+b_{2}\right)
$$

and

$$
\left(a_{1} \oplus b_{1}\right)\left(a_{2} \oplus b_{2}\right)=a_{1} a_{2} \oplus\left(a_{1} b_{2}+a_{2} b_{1}\right) .
$$

Let $M$ be an $R$-module such that $M=A \oplus A$ as an abelian group and

$$
(a \oplus b)(x \oplus y)=a x \oplus\left(a y+t^{d} b x\right)
$$

for $a \oplus b \in R$ and $x \oplus y \in M$.

Then

$$
\operatorname{Ext}_{R}^{i}(M, M) \cong k^{2 d}
$$

for $i>0$.

Proof. Let $I=\left\{(a \oplus b) \in R \mid a / t^{d} \in A\right\}$. Then $I$ is an ideal of $R$. Let $\phi$ be a map from $M$ to $I$ defined by $\phi(x \oplus y)=t^{d} x \oplus y$. It is easy to verify that $\phi$ is an isomorphism of $R$-modules. Define a homomorphism

$$
f_{0}: R^{2} \rightarrow I,\left(a_{1} \oplus b_{1}, a_{2} \oplus b_{2}\right) \mapsto t^{d} a_{1} \oplus\left(t^{d} b_{1}-a_{2}\right) .
$$


Then $f_{0}$ is surjective and $\operatorname{Ker}\left(f_{0}\right)=\left\{\left(0 \oplus b_{1}, a_{2} \oplus b_{2}\right) \mid a_{2}=t^{d} b_{1}\right\}$. Define a surjective homomorphism

$$
f_{1}: R^{2} \rightarrow \operatorname{Ker}\left(f_{0}\right),\left(a_{1} \oplus b_{1}, a_{2} \oplus b_{2}\right) \mapsto\left(0 \oplus a_{1}, a_{1} t^{d} \oplus b_{1} t^{d}-a_{2}\right) .
$$

It is obvious that $\operatorname{Ker}\left(f_{1}\right)=\operatorname{Ker}\left(f_{0}\right)$. Thus we have a free resolution

$$
\cdots \stackrel{f_{1}}{\rightarrow} R^{2} \stackrel{f_{1}}{\rightarrow} R^{2} \stackrel{f_{1}}{\rightarrow} R^{2} \stackrel{f_{0}}{\rightarrow} I \rightarrow 0 .
$$

Let $g: \operatorname{Hom}_{R}\left(R^{2}, I\right) \rightarrow \operatorname{Hom}_{R}\left(R^{2}, I\right), h \mapsto h f_{1}$. Then $\operatorname{Ext}_{R}^{i}(I, I) \cong \operatorname{Ker}(g) /$ $\operatorname{Im}(g)$ for $i>0$. If we identify $\operatorname{Hom}_{R}\left(R^{2}, I\right)$ with $I^{2}$ in the canonical way, then

$$
\operatorname{Ker}(g)=\left\{\left(t^{d} x_{1} \oplus y_{1}, 0 \oplus\left(-x_{1}\right)\right) \mid x_{1}, y_{1} \in A\right\}
$$

and

$$
\operatorname{Im}(g)=\left\{\left(t^{2 d} x_{2} \oplus\left(t^{d} y_{2}+t^{d} x_{1}\right), 0 \oplus\left(-t^{d} x_{2}\right)\right) \mid x_{1}, x_{2}, y_{2} \in A\right\}
$$

by direct computation. Thus $\operatorname{Ker}(g) / \operatorname{Im}(g) \cong k^{2 d}$ and the proof of the Sublemma concludes.

Thus far the proof of Lemma 4.1 is complete.

Corollary 4.3. Let $d=\operatorname{deg}\left(\mathcal{L}^{\prime}\right)-\operatorname{deg}(\mathcal{M})-\operatorname{deg}\left(\mathcal{L}^{\prime \prime}\right)$. Then

$$
H^{0}\left(\mathcal{E} x t_{\mathcal{O}_{C^{\prime}}}^{i}(\mathcal{F}, \mathcal{F})\right) \cong k^{2 d}
$$

and

$$
H^{j}\left(\mathcal{E} x t_{\mathcal{O}_{C^{\prime}}}^{i}(\mathcal{F}, \mathcal{F})\right)=0
$$

for $i>0$ and $j>0$.

Proof. Since $\mathcal{E} x t_{\mathcal{O}_{C^{\prime}}}^{i}(\mathcal{F}, \mathcal{F})_{z} \cong \operatorname{Ext}_{\mathcal{O}_{C^{\prime}, z}}^{i}\left(\mathcal{F}_{z}, \mathcal{F}_{z}\right)$ for any point $z \in C$, the lemma implies that $\mathcal{E} x t_{\mathcal{O}_{C^{\prime}}}^{i}(\mathcal{F}, \mathcal{F})$ is supported on $D$ for $i>0$.

THEOREM 4.4. Let $\mathcal{F}$ be a pure sheaf of rank 2 on $C^{\prime}$ such that $\mathcal{F}^{0} \cong \mathcal{L}^{\prime}$ and $\mathcal{F} / \mathcal{F}^{0} \cong \mathcal{L}^{\prime \prime}$. Let $d=\operatorname{deg}\left(\mathcal{L}^{\prime}\right)-\operatorname{deg}(\mathcal{M})-\operatorname{deg}\left(\mathcal{L}^{\prime \prime}\right)$. Then

1) $\operatorname{Ext}_{\mathcal{O}_{C^{\prime}}}^{i}(\mathcal{F}, \mathcal{F}) \cong k^{2 d}$ for $i>1$.

2) $\operatorname{dim}_{k}\left(\operatorname{Ext}_{\mathcal{O}_{C^{\prime}}}^{1}(\mathcal{F}, \mathcal{F})\right)=2 d+g(C)+\operatorname{dim}_{k}\left(H^{1}\left(C, \mathcal{L}^{\prime \prime-1} \otimes \mathcal{L}^{\prime}\right)\right)$.

Proof. Take spectral sequence

$$
E_{2}^{p, q}=H^{p}\left(\mathcal{E} x t_{\mathcal{O}_{C^{\prime}}}^{q}(\mathcal{F}, \mathcal{F})\right)
$$

Then we have

$$
E_{2}^{p, q} \Rightarrow \operatorname{Ext}_{\mathcal{O}_{C^{\prime}}}^{\bullet}(\mathcal{F}, \mathcal{F})
$$


By Corollary $4.3 E_{2}^{p, q}=0$ for all $p>0, q>0$. Hence

$$
\operatorname{Ext}_{\mathcal{O}_{C^{\prime}}}^{i}(\mathcal{F}, \mathcal{F}) \cong H^{0}\left(\mathcal{E} x t_{\mathcal{O}_{C^{\prime}}}^{i}(\mathcal{F}, \mathcal{F})\right) \cong k^{2 d}
$$

for $i>1$.

Since $H^{2}\left(\mathcal{H o m}_{\mathcal{O}_{C^{\prime}}}(\mathcal{F}, \mathcal{F})\right)=0$, we have a short exact sequence

$$
0 \rightarrow H^{1}\left(\mathcal{H o m}{\mathcal{O}_{C^{\prime}}}(\mathcal{F}, \mathcal{F})\right) \rightarrow \operatorname{Ext}_{\mathcal{O}_{C^{\prime}}}^{1}(\mathcal{F}, \mathcal{F}) \rightarrow H^{0}\left(\mathcal{E} x t_{\mathcal{O}_{C^{\prime}}}^{1}(\mathcal{F}, \mathcal{F})\right) \rightarrow 0
$$

The short exact sequence

$$
0 \rightarrow \mathcal{H o m}_{C}\left(\mathcal{L}^{\prime \prime}, \mathcal{L}^{\prime}\right) \rightarrow \mathcal{H o m}_{C^{\prime}}(\mathcal{F}, \mathcal{F}) \rightarrow \mathcal{O}_{C} \rightarrow 0
$$

implies that

$$
\operatorname{dim}_{k}\left(H^{1}\left(\mathcal{H} o m_{C^{\prime}}(\mathcal{F}, \mathcal{F})\right)\right)=\operatorname{dim}_{k}\left(H^{1}\left(\mathcal{H} o m_{C}\left(\mathcal{L}^{\prime \prime}, \mathcal{L}\right)\right)\right)+g(C) .
$$

It follows from $(8),(9)$ and Corollary 4.3 that $\operatorname{dim}_{k}\left(\operatorname{Ext}_{\mathcal{O}_{C^{\prime}}}^{1}(\mathcal{F}, \mathcal{F})\right)=2 d+g(C)+$ $\operatorname{dim}_{k}\left(H^{1}\left(C, \mathcal{L}^{\prime \prime-1} \otimes \mathcal{L}^{\prime}\right)\right)$.

\section{Coherent sheaves of rank 2 over a fat $\mathbb{P}^{1}$}

Let $C=\mathbb{P}^{1}$ be the the projective line over an algebraically closed field $k$. Let $d$ be a fixed integer. Let $C^{\prime}$ be an infinitesimal extension of $C$ by $\mathcal{O}(-d)$.

According to Lemma 3.1 there is an invertible sheaf $\mathcal{L}^{\prime}$ on $C^{\prime}$ such that $\mathcal{L}^{\prime} \otimes$ $\mathcal{O}_{C} \cong \mathcal{O}(1)$. Such a sheaf is not necessarily unique, we choose one and denote it by $\mathcal{O}_{C^{\prime}}(1)$. The $n$-th power of this sheaf is denoted by $\mathcal{O}_{C^{\prime}}(n)$.

LemMa 5.1. If $d \leq 0, n>0$ or $d>0, n \geq d$, then the invertible sheaf $\mathcal{O}_{C^{\prime}}(n)$ is very ample.

Proof. Let $p, q$ be two closed points of $C^{\prime}$. Let $\mathfrak{m}_{p}$ and $\mathfrak{m}_{q}$ be the maximal ideals of the local rings $\mathcal{O}_{C, p}$ and $\mathcal{O}_{C, q}$ respectively. Let $\mathfrak{m}_{p}^{\prime}$ and $\mathfrak{m}_{q}^{\prime}$ be the maximal ideals of the local rings $\mathcal{O}_{C^{\prime}, p}$ and $\mathcal{O}_{C^{\prime}, q}$ respectively. Then $\mathfrak{m}_{p}^{\prime}=\mathfrak{m}_{p} \oplus \mathcal{O}_{C, p}$ and $\mathfrak{m}_{q}^{\prime}=\mathfrak{m}_{q} \oplus \mathcal{O}_{C, q}$.

There is an exact sequence

$$
0 \rightarrow \mathcal{O}_{C}(n-d) \rightarrow \mathcal{O}_{C^{\prime}}(n) \rightarrow \mathcal{O}_{C}(n) \rightarrow 0
$$

Since $n-d \geq 0$, we have $H^{1}\left(C, \mathcal{O}_{C}(n-d)\right)=0$ and an exact sequence

$$
0 \rightarrow H^{0}\left(\mathcal{O}_{C}(n-d)\right) \rightarrow H^{0}\left(\mathcal{O}_{C^{\prime}}(n)\right) \rightarrow H^{0}\left(\mathcal{O}_{C}(n)\right) \rightarrow 0
$$


Since $n>0$, there exists $s_{1} \in \Gamma\left(C, \mathcal{O}_{C}(n)\right)$ such that $s_{1} \in \mathfrak{m}_{p} \mathcal{O}_{C}(n)_{p}$ and $s_{1} \notin$ $\mathfrak{m}_{q} \mathcal{O}_{C}(n)_{q}$. Choose some $s \in H^{0}\left(\mathcal{O}_{C^{\prime}}(n)\right)$ whose image in $H^{0}(\mathcal{C}(n))$ is $s_{1}$. Then $s \in \mathfrak{m}_{p}^{\prime} \mathcal{O}_{C^{\prime}}(n)_{p}$ and $s \notin \mathfrak{m}_{q}^{\prime} \mathcal{O}_{C^{\prime}}(n)_{q}$.

In a similar manner one can show that the set $\left\{s \in \Gamma\left(C^{\prime}, \mathcal{O}_{C^{\prime}}(n)\right) \mid s \in\right.$ $\left.\mathfrak{m}_{p}^{\prime} \mathcal{O}_{C^{\prime}}(n)_{p}\right\}$ spans $\mathfrak{m}_{p}^{\prime} \mathcal{O}_{C^{\prime}}(n)_{p} / \mathfrak{m}^{\prime 2} \mathcal{O}_{C^{\prime}}(n)_{p}$. This concludes the proof of the lemma.

From now on, we fix a very ample sheaf $\mathcal{H}=\mathcal{O}_{C^{\prime}}(n)$ on $C^{\prime}$, where $n=1$ if $d \leq 0$ and $n=d$ if $d>0$.

Definition 5.2. Let $\mathcal{F}$ be a pure sheaf on $C^{\prime}$. The Hilbert polynomial $P(\mathcal{F})$ is defined to be the numerical polynomial such that

$$
P(\mathcal{F})(n)=h^{0}\left(C^{\prime}, \mathcal{F} \otimes \mathcal{H}^{n}\right)
$$

for sufficiently large $n$. Let $r$ be the leading coefficient of $P(\mathcal{F})$. The reduced Hilbert polynomial $p(\mathcal{F})$ is defined to be

$$
p(\mathcal{F})=\frac{P(\mathcal{F})}{r} .
$$

Lemma 5.3. Let $\mathcal{F}$ be a pure sheaf of rank 2 on $C^{\prime}$ such that $[\mathcal{F}] \in$ $M\left(\mathcal{O}_{\mathbb{P}_{k}^{1}}(a), \mathcal{O}_{\mathbb{P}_{k}^{1}}(b)\right)$. Then $P(\mathcal{F})(n)=2 n+a+b+2$ if $d \leq 0$ and $P(\mathcal{F})(n)=$ $2 d n+a+b+2$ if $d>0$.

Proof. We only settle the case $d>0$. The other case is verified by the same method.

Tensoring the exact sequence

$$
0 \rightarrow \mathcal{O}_{\mathbb{P}_{k}^{1}}(b) \rightarrow \mathcal{F} \rightarrow \mathcal{O}_{\mathbb{P}_{k}^{1}}(a) \rightarrow 0
$$

by $\mathcal{H}^{n}$ we obtain an exact sequence

$$
0 \rightarrow \mathcal{O}_{\mathbb{P}_{k}^{1}}(n d+b) \rightarrow \mathcal{F} \otimes \mathcal{H}^{n} \rightarrow \mathcal{O}_{\mathbb{P}_{k}^{1}}(n d+a) \rightarrow 0
$$

Hence

$$
h^{0}\left(C^{\prime}, \mathcal{F} \otimes \mathcal{H}^{n}\right)=h^{0}\left(\mathbb{P}_{k}^{1}, \mathcal{O}_{\mathbb{P}_{k}^{1}}(n d+a)\right)+h^{0}\left(\mathbb{P}_{k}^{1}, \mathcal{O}_{\mathbb{P}_{k}^{1}}(n d+b)\right)=2 n d+a+b+2
$$

for sufficiently large $n$.

Definition 5.4. Let $\mathcal{F}$ be a pure sheaf on $C^{\prime}$. If $p(\mathcal{E}) \leq p(\mathcal{F})$ for every non-zero coherent subsheaf $\mathcal{E}$ of $\mathcal{F}$ then $\mathcal{F}$ is semistable. If $p(\mathcal{E})<p(\mathcal{F})$ for every non-trivial coherent subsheaf $\mathcal{E}$ of $\mathcal{F}$ then $\mathcal{F}$ is stable. If $\mathcal{F}$ is semistable but not stable then it is called strictly semistable. 
Proposition 5.5. If $d \leq 0$, then every pure sheaf $\mathcal{F}$ of rank 2 is not stable.

Proof. Since every locally free sheaf on $\mathbb{P}^{1}$ is decomposable, any pure sheaf of rank 2 on $C^{\prime}$ of type II is not stable. Suppose that the sheaf $\mathcal{F}$ in question. is stable. Then $\mathcal{F}^{0} \cong \mathcal{O}_{C}(m)$ and $\mathcal{F} / \mathcal{F}^{0} \cong \mathcal{O}_{C}(n)$ for some integers $m$ and $n$ with $m<n$. This would imply that $\operatorname{Hom}_{\mathcal{O}_{\mathbb{p} 1}}(\mathcal{O}(n-d), \mathcal{O}(m))=0$, contradicting Theorem 3.5.

Definition 5.6. Let $\mathcal{E}$ be a semistable coherent sheaf on a $C^{\prime}$. Let

$$
0=\mathcal{E}_{0} \subset \mathcal{E}_{1} \subset \cdots \subset \mathcal{E}_{r}=\mathcal{E}
$$

be a filtration such that the quotients $\mathcal{E}_{i} / \mathcal{E}_{i-1}$ are stable with the same normalized Hilbert polynomials. Let $\operatorname{gr}(\mathcal{E})=\oplus \mathcal{E}_{i} / \mathcal{E}_{i-1}$. Let $\mathcal{E}$ and $\mathcal{F}$ be two semistable coherent sheaves on $C^{\prime}$. If $\operatorname{gr}(\mathcal{E}) \cong g r(\mathcal{F})$ then we say that $\mathcal{E}$ and $\mathcal{F}$ are equivalent.

LEMma 5.7. Let $d$ be a positive integer. Let $C^{\prime}$ be an infinitesimal extension of $C=\mathbb{P}^{1}$ by $\mathcal{O}(-d)$. Let $P(n)=2 d n+m+2$ be a numerical polynomial in $n$. If $m$ is odd, then there is no strictly semistable pure sheaf of rank 2 on $C^{\prime}$ with Hilbert polynomial $P(n)$. If $m$ is even, then there is exactly one equivalent class of strictly semistable pure sheaves of rank 2 with Hilbert polynomial $P(n)$.

Proof. Let $\mathcal{F}$ be a strictly semistable pure sheaf of rank 2 with Hilbert polynomial $P(n)$. Then there is an exact sequence

$$
0 \rightarrow \mathcal{O}_{\mathbb{P}^{1}}(a) \rightarrow \mathcal{F} \rightarrow \mathcal{O}_{\mathbb{P}^{1}}(a) \rightarrow 0
$$

for some integer $a$. Then the Hilbert polynomial of $\mathcal{F}$ is $2 d n+2 a+2$ by Lemma 5.3. Hence $m=2 a$ is even. Since $g r(\mathcal{F}) \cong \mathcal{O}_{\mathbb{P}^{1}}(a) \oplus \mathcal{O}_{\mathbb{P}^{1}}(a)$, which is totally determined by the Hilbert polynomial $P(n)$, all strictly semistable pure sheaves of rank 2 with Hilbert polynomial $P(n)$ are equivalent.

Let $m$ be an integer. Let $P(n)=2 d n+m+2$. It is known that the coarse moduli of semistable pure sheaves of rank 2 on $C^{\prime}$ with Hilbert polynomial $P(n)$ exists (cf. Theorem 1.21 of [7]) and it is a projective scheme. Let us denote it by $\mathfrak{M}(m)_{s s}$. Its open subscheme consisting of all stable sheaves is denoted by $\mathfrak{M}(m)_{s}$. Let $\mathfrak{M}(m)_{s s, \text { red }}$ and $\mathfrak{M}(m)_{s, \text { red }}$ denote the corresponding reduced induced schemes.

TheOREM 5.8. Let $\left(a_{1}, b_{1}\right),\left(a_{2}, b_{2}\right), \ldots,\left(a_{r}, b_{r}\right)$ be the set of all pairs of integers such that $0<a_{i}-b_{i} \leq d$ and $a_{i}+b_{i}=m$. Then

1) $\mathfrak{M}(m)_{s, \text { red }}$ is the disjoint union of $r$ irreducible smooth varieties $Y_{i}(1 \leq$ $i \leq r)$ of dimension $d-1$.

2) Every point in $Y_{i}$ represents a member of $M\left(\mathcal{O}_{\mathbb{P}^{1}}\left(a_{i}\right), \mathcal{O}_{\mathbb{P}^{1}}\left(b_{i}\right)\right)$.

3) If $a_{i}-b_{i}=d$ then $\mathfrak{M}(m)_{s}$ is smooth on $Y_{i}$, otherwise $\mathfrak{M}(m)_{s}$ is not reduced along $Y_{i}$. 
4) If $m$ is odd, then $\mathfrak{M}(m)_{s}=\mathfrak{M}(m)_{\text {ss }}$, otherwise $\mathfrak{M}(m)_{s s} \backslash \mathfrak{M}(m)_{s}$ is a single point representing the class of strictly semistable sheaves.

5) Let $\bar{Y}_{i}$ be the closure of $Y_{i}$ in $\mathfrak{M}(m)_{\text {ss }}$. Then $\bar{Y}_{1}, \ldots, \bar{Y}_{r}$ are all irreducible components of $\mathfrak{M}(m)_{s s}$.

Proof. Let $\mathcal{F}$ be a rank 2 stable pure sheaf on $C^{\prime}$ with Hilbert polynomial $P(n)$. Then $\mathcal{F}$ is of type I. Assume that $\mathcal{F}^{0} \cong \mathcal{O}_{\mathbb{P}^{1}}(b)$ and $\mathcal{F} / \mathcal{F}^{0} \cong \mathcal{O}_{\mathbb{P}^{1}}(a)$. Then $a>b$ by stability. By Lemma 5.3 we have $a+b=m$. By Theorem $3.5 a, b$ satisfy the condition $H^{0}\left(\mathbb{P}^{1}, \mathcal{O}(d-a+b)\right) \neq 0$. Hence $d \geq a-b$. Hence

$$
M\left(\mathcal{O}_{\mathbb{P}^{1}}\left(a_{1}\right), \mathcal{O}_{\mathbb{P}^{1}}\left(b_{1}\right)\right), \ldots, M\left(\mathcal{O}_{\mathbb{P}^{1}}\left(a_{r}\right), \mathcal{O}_{\mathbb{P}^{1}}\left(b_{r}\right)\right)
$$

form a stratum of $\mathfrak{M}(m)_{s, \text { red }}$. Identify $Y_{i}$ with $M\left(\mathcal{O}_{\mathbb{P}^{1}}\left(a_{i}\right), \mathcal{O}_{\mathbb{P}^{1}}\left(b_{i}\right)\right)$. Proposition 3.7 implies that each $Y_{i}$ is a smooth quasi-projective variety of dimension $d-1$. Thus 1) and 2) have been proved.

Since all $Y_{i}$ have the same dimension $d-1, Y_{i}$ is not contained in $\bigcup_{j \neq i} \bar{Y}_{j}$. Hence 5) holds.

If $a_{i}-b_{i}=d$, then $\operatorname{dim}_{k}\left(\operatorname{Ext}_{C^{\prime}}^{1}(\mathcal{F}, \mathcal{F})\right)=d-1=\operatorname{dim}\left(Y_{i}\right)$ and $\operatorname{Ext}_{C^{\prime}}^{2}(\mathcal{F}, \mathcal{F})=0$ by Theorem 4.4. By deformation theory, $\mathfrak{M}(m)_{s}$ is non-singular at $[\mathcal{F}]$. If $a_{i}-b_{i}>d$, then $\operatorname{dim}_{k}\left(\operatorname{Ext}_{C^{\prime}}^{1}(\mathcal{F}, \mathcal{F})\right)>d-1$ by Theorem 4.4. But $Y_{i}$ is smooth at every point. This means that $\mathfrak{M}(m)_{s}$ is not reduced along $Y_{i}$. This proves 3$)$.

The statement 4) follows from Lemma 5.7.

REMARK 5.9. If $a_{i}-b_{i}=d$ then $Y_{i}$ represents all invertible sheaf with Hilbert polynomial $P(n)$ on $C^{\prime}$. Hence we may define $Y_{i}$ to be the generalized Jacobian $J a c\left(C^{\prime}\right)$ of the fat curve $C^{\prime}$. By Theorem $3.5 J a c\left(C^{\prime}\right) \cong \mathbb{A}_{k}^{d-1}$. It is natural to define $\bar{Y}_{i}$ to be the compactification of $\operatorname{Jac}\left(C^{\prime}\right)$.

We now take a look at the structure of the moduli scheme for small $d$.

1) $d=1:$

In this case $\mathfrak{M}(m)_{s s}$ is a single point. If $m$ is even then $\mathfrak{M}(m)_{s}$ is empty. If $m$ is odd, then $\mathfrak{M}(m)_{s s}=\mathfrak{M}(m)_{s}$ represents the unique invertible sheaf whose Hilbert polynomial is $2 n+m+2$.

2) $d=2$ :

If $m=2 t$ for some integer $t$, then the only pair of integers $(a, b)$ satisfying $a+b=m$ and $0<a-b \leq 2$ is $(t+1, t-1)$. Theorem 5.8 and Theorem 3.5 imply that $\mathfrak{M}(m)_{s} \cong \mathbb{A}^{1}$. Thus $\mathfrak{M}(m)_{s s, r e d}(m)$ is a rational curve.

If $m=2 t+1$ for some integer $t$, then the only pair of integers $(a, b)$ satisfying $a+b=m$ and $0<a-b \leq 2$ is $(t+1, t)$. Theorem 5.8 and Lemma 5.7 imply that $\mathfrak{M}(m)_{s s, r e d}=\mathfrak{M}(m)_{s}=M\left(\mathcal{O}_{\mathbb{P}^{1}}(t+1), \mathcal{O}_{\mathbb{P}^{1}}(t)\right)$ which is isomorphic to $\mathbb{P}^{1}$ by Theorem 3.5. 
According to Theorem $5.8 \mathfrak{M}(m)_{s s}$ is not reduced. Let us investigate it's structure by taking $m=-3$. Every member $\mathcal{E}$ of $M\left(\mathcal{O}_{\mathbb{P}^{1}}(-1), \mathcal{O}_{\mathbb{P}^{1}}(-2)\right)$ determines a commutative diagram

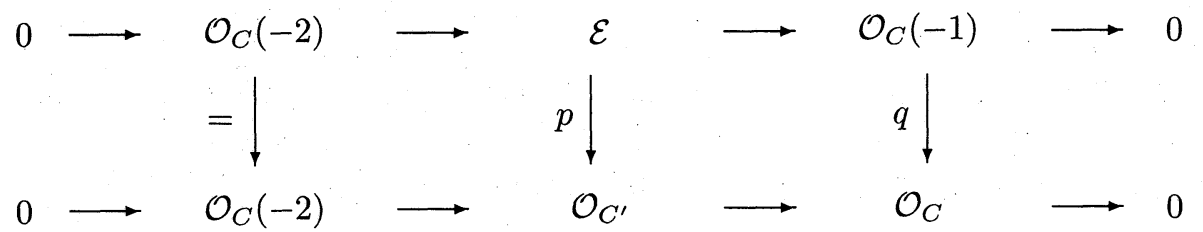

where $p$ and $q$ are injective. Moreover, the functor of flat families of pure sheaves with Hilbert polynomial $P(n)=4 n-1$ is equivalent to the functor of quotient schemes of $\mathcal{O}_{C^{\prime}}$ with Hilbert polynomial 1 . The latter is represented by $\mathbf{H i l b}_{C^{\prime}}^{1} \cong$ $C^{\prime}$. In other words, $\mathfrak{M}(m)_{s} \cong C^{\prime}$ is a fine moduli.

3) $d=3$ :

Case i) $m$ is even.

In this case there is only one pair of integer $(a, b)$ such that $a+b=m$ and $0<a-b \leq 3$. That is $a=m / 2+1, b=m / 2-1$. Theorem 5.8 implies that $\mathfrak{M}(m)_{s, \text { red }} \cong M\left(\mathcal{O}_{C}(m / 2+1), \mathcal{O}_{C}(m / 2-1)\right)$, which is isomorphic to $\mathbb{P}\left(H^{1}\left(C, \mathcal{O}_{C}(-2)\right) \times H^{0}\left(C, \mathcal{O}_{C}(1)\right)^{*}\right)$ by Theorem 3.5. Hence $\mathfrak{M}(m)_{s, r e d}$ is isomorphic to $\mathbb{P}^{2} \backslash\{p\}$, where $p$ is a point. Therefore $\mathfrak{M}(m)_{s s}$ is an irreducible surface birational to $\mathbb{P}^{2}$ by Lemma 5.7. The space of first order deformations has dimension 3 by Theorem 4.4 .

Case ii) $m$ is odd.

This is an interesting case. According to Theorem 5.8 the space $\mathfrak{M}(m)_{\text {ss, red }}$ consists of two irreducible components.

By Remark 3.4 we may assume that $m=-3 . \mathfrak{M}(m)_{s s, \text { red }}=\mathfrak{M}(m)_{s, \text { red }}$ is the disjoint union of $J a c\left(C^{\prime}\right) \cong \mathbb{A}^{2}$ and $M(\mathcal{O}(-1), \mathcal{O}(-2)) \cong \mathbb{P}^{2}$. Since $\mathfrak{M}(m)_{s s}$ is projective, the closure of $J a c\left(C^{\prime}\right)$ has a non-empty intersection with $M(\mathcal{O}(-1), \mathcal{O}(-2))$, which turns out to be a conic in $M(\mathcal{O}(-1), \mathcal{O}(-2))$, as we shall see.

LEMMA 5.10. Every infinitesimal extension of $\mathbb{P}^{1}$ by $\mathcal{O}(-3)$ is trivial.

Proof. Since $H^{1}\left(\mathbb{P}^{1}, \mathcal{H}\right.$ om $\left.\left(\Omega_{\mathbb{P}^{1}}, \mathcal{O}(-3)\right)\right) \cong H^{1}\left(\mathbb{P}^{1}, \mathcal{O}(-1)\right)=0$, the lemma follows from Lemma 2.3.

Now let $C^{\prime}$ denote the infinitesimal extension of $C=\mathbb{P}^{1}$ by $\mathcal{O}(-3)$. Let $\mathcal{F}$ be a rank 2 pure sheaf such that $\mathcal{F}^{0} \cong \mathcal{O}(-2)$ and $\mathcal{F} / \mathcal{F}^{0} \cong \mathcal{O}(-1)$. Let $s$ be the point in $\mathfrak{M}(-3)_{s}$ representing $\mathcal{F}$. We want to decide under what condition $s$ is in the boundary of $J a c\left(C^{\prime}\right)$. That means we need to know under what condition $\mathcal{F}$ can be deformed into an invertible sheaf on $C^{\prime}$. 
Since $C^{\prime}$ is a trivial extension by Lemma 5.10, we may write $\mathcal{O}_{C^{\prime}}=\mathcal{O}_{C} \oplus$ $\mathcal{O}_{C}(-3)$.

By Theorem $3.5 M(\mathcal{O}(-1), \mathcal{O}(-2)) \cong \mathbb{P}\left(H^{0}\left(\mathbb{P}^{1}, \mathcal{O}(2)\right)\right) \cong \mathbb{P}^{2}$. Let $U_{0}=$ $\operatorname{Spec}(k[x])$ and $U_{1}=\operatorname{Spec}(k[y])$ be a standard open covering of $\mathbb{P}^{1}$ with $x y=1$ on $U_{0} \cap U_{1}$. Then $H^{0}\left(U_{0}, \mathcal{O}_{C^{\prime}}\right) \cong k[x]\left[\epsilon_{0}\right]$ and $H^{0}\left(U_{1}, \mathcal{O}_{C^{\prime}}\right) \cong k[y]\left[\epsilon_{1}\right]$ such that $\epsilon_{0}^{2}=\epsilon_{1}^{2}=0$ and $\epsilon_{0}=\epsilon_{1} / y^{3}$ on $U_{0} \cap U_{1}$.

Let $\left(a_{0}: a_{1}: a_{2}\right) \in \mathbb{P}^{2}$. Let $Q_{0}=k[x] \oplus k[x]$ and $Q_{1}=k[y] \oplus k[y]$. Define a $k[x]\left[\epsilon_{0}\right]$-module structure on $Q_{0}$ by $\left(f(x)+g(x) \epsilon_{0}\right)(a(x) \oplus b(x))=f(x) a(x) \oplus$ $\left[f(x) b(x)+g(x) a(x)\left(a_{0}+a_{1} x+a_{2} x^{2}\right)\right]$. Similarly define a $k[x]\left[\epsilon_{1}\right]$-module structure on $Q_{1}$ by $\left(f(y)+g(y) \epsilon_{1}\right)(a(y) \oplus b(y))=f(y) a(y) \oplus\left[f(y) b(y)+g(y) a(y)\left(a_{0} y^{2}+\right.\right.$ $\left.\left.a_{1} y+a_{2} y^{2}\right)\right]$. Then glue the sheaves $\tilde{Q}_{0}$ on $U_{0}$ and $\tilde{Q}_{1}$ on $U_{1}$ by $a(x) \oplus b(x)=$ $a(1 / y) / y \oplus b(1 / y) / y^{2}$ on $U_{0} \cap U_{1}$. Then we obtain a sheaf on $C^{\prime}$, denoted by $\mathcal{F}\left(a_{0}: a_{1}: a_{2}\right)$. When $\left(a_{0}: a_{1}: a_{2}\right)$ runs over $\mathbb{P}^{2}$ the class $\left[\mathcal{F}\left(a_{0}: a_{1}: a_{2}\right)\right]$ runs through all members of $M(\mathcal{O}(-1), \mathcal{O}(-2))$.

LeMma 5.11. Let $\left(a_{0}: a_{1}: a_{2}\right) \in \mathbb{P}^{2}$.

1) If $a_{1}^{2}=4 a_{0} a_{2}$, then there exists a flat family of sheaves on $C^{\prime}$ over a variety $S$ of positive-dimension and a closed point $s \in S$ such that the fiber at $s$ is isomorphic to $\mathcal{F}\left(a_{0}: a_{1}: a_{2}\right)$ and the fibers elsewhere are invertible sheaves on $C^{\prime}$.

2). If $a_{1}^{2} \neq 4 a_{0} a_{2}$, then for any flat family of sheaves over a reduced variety $S$ with a closed point $s \in S$ such that the fiber at $s$ is isomorphic to $\mathcal{F}\left(a_{0}: a_{1}: a_{2}\right)$ there exists an open neighborhood $V$ of $s$ in $S$ such that every fiber over $V$ is in $M(\mathcal{O}(-1), \mathcal{O}(-2))$.

Proof. 1) Let $S=\mathbb{A}^{1}=\operatorname{Spec}(k[t])$ and let $s$ be the zero point. Let $X^{\prime}=C^{\prime} \times S$ and $X=\mathbb{P}_{k}^{1} \times S$. The underlying spaces of $X^{\prime}$ and $X$ are the same. Let $\pi_{1}: X \rightarrow \mathbb{P}_{k}^{1}$ and $\pi_{2}: X \rightarrow S$ be the projection maps. Let $\mathcal{G}=\pi_{1}^{*}(\mathcal{O}(-1) \oplus \mathcal{O}(-2))$. Then $\mathcal{G}$ is a locally free sheaf on $X$ of rank 2 such that $H^{0}\left(U_{0} \times S, \mathcal{G}\right) \cong k[x, t] \oplus k[x, t]$ and $H^{0}\left(U_{1} \times S, \mathcal{G}\right) \cong k[y, t] \oplus k[y, t]$ with the transition tranform

$$
a(x, t) \oplus b(x, t)=a(1 / y, t) / y \oplus b(1 / y, t) / y^{2} .
$$

Since $a_{1}^{2}=4 a_{0} a_{2}$, there exists a pair of constants $\left(b_{0}, b_{1}\right) \neq(0,0)$ such that $a_{0} u_{0}^{2}+a_{1} u_{0} u_{1}+a_{2}=\left(b_{0} u_{0}+b_{1} u_{1}\right)^{2}$. Choose $c_{0}, c_{1} \in k$ such that $c_{0} b_{1}-c_{1} b_{0} \neq 0$.

In order to define a $k[x, t]\left[\epsilon_{0}\right]$-module structure on $k[x, t] \oplus k[x, t]$ we need to define the multiplications of $\epsilon_{0}$ with $1 \oplus 0$ and $0 \oplus 1$, which are determined by a $2 \times 2$ matrix with entries in $k[x, t]$. Since $\epsilon_{0}^{2}=0$, the square of this matrix must be zero. We take the matrix

$$
\left(\begin{array}{cc}
t\left(b_{0}+b_{1} x\right)\left(c_{0}+c_{1} x\right)^{2} & \left(b_{0}+b_{1} x\right)^{2} \\
-t^{2}\left(c_{0}+c_{1} x\right)^{4} & -t\left(b_{0}+b_{1} x\right)\left(c_{0}+c_{1} x\right)^{2}
\end{array}\right) .
$$


More precisely, we put a $k[x, t]\left[\epsilon_{0}\right]$-module structure on $H^{0}\left(U_{0} \times S, \mathcal{G}\right)$ and a $k[y, t]\left[\epsilon_{1}\right]$-module structure on $H^{0}\left(U_{1} \times S, \mathcal{G}\right)$ as follows:

$$
\begin{aligned}
\left(f+g \epsilon_{0}\right)(p \oplus q)= & \left(f p+t\left(b_{0}+b_{1} x\right)\left(c_{0}+c_{1} x\right)^{2} g p-t^{2}\left(c_{0}+c_{1} x\right)^{4} g q\right) \\
& \oplus\left(f q+\left(b_{0}+b_{1} x\right)^{2} g p-t\left(b_{0}+b_{1} x\right)\left(c_{0}+c_{1} x\right)^{2} g q\right) \\
\left(f+g \epsilon_{1}\right)(p \oplus q)= & \left(f p+t\left(b_{0} y+b_{1}\right)\left(c_{0} y+c_{1}\right)^{2} g p-t^{2}\left(c_{0} y+c_{1}\right)^{4} g q\right) \\
& \oplus\left(f q+\left(b_{0} y+b_{1}\right)^{2} g p-t\left(b_{0} y+b_{1}\right)\left(c_{0} y+c_{1}\right)^{2} g q\right) .
\end{aligned}
$$

It can be verified that these two module structures are compatible with the transition transform (11). Hence $\mathcal{G}$ becomes a sheaf on $X^{\prime}$, flat over $S$.

Obviously $\mathcal{G}_{0} \cong \mathcal{F}\left(a_{0}: a_{1}: a_{2}\right)$. We claim that every $\mathcal{G}_{t}$ for $t \neq 0$ is an invertible sheaf on $C^{\prime}$.

For any fixed $t \neq 0$, we have $H^{0}\left(U_{0}, \mathcal{G}_{t}^{0}\right)=k[x] e_{0}$ and $H^{0}\left(U_{1}, \mathcal{G}_{t}^{0}\right)=k[y] e_{1}$, where $e_{0}=t\left(c_{0}+c_{1} x\right)^{2} \oplus\left(b_{0}+b_{1} x\right)$ and $e_{1}=t\left(c_{0} y+c_{1}\right)^{2} \oplus\left(b_{0} y+b_{1}\right)$. Since $e_{0}=e_{1} / y^{3}$, we conclude that $\mathcal{G}_{t}^{0} \cong \mathcal{O}(-3)$, which implies that $\left[\mathcal{G}_{t}^{0}\right] \in \mathfrak{M}(\mathcal{O}, \mathcal{O}(-3))$. Therefore $\mathcal{G}_{t}$ is an invertible sheaf on $C^{\prime}$.

2) Let $\mathcal{G}$ be a coherent sheaf on $X^{\prime}=C^{\prime} \times S$ flat over $S$. Assume that $\mathcal{G}_{s} \cong \mathcal{F}\left(a_{0}: a_{1}: a_{2}\right)$ with $a_{1}^{2}-4 a_{0} a_{2} \neq 0$.

Regard $\mathcal{G}$ as a sheaf on $X=C \times S$. By the flatness every fiber of $\mathcal{G}$ is isomorphic to $\mathcal{O}_{C}(-1) \oplus \mathcal{O}_{C}(-2)$ as an $\mathcal{O}_{C}$ module. Hence there is an open neighborhood $V$ of $s$ such that $\mathcal{G} \cong \pi_{1}^{*}\left(\mathcal{O}_{C}(-1) \oplus \mathcal{O}_{C}(-2)\right)$ as an $\mathcal{O}_{C \times V}$-module. Without loss of generality we may assume that $V=S=\operatorname{Spec}(A)$ is an affine variety.

Similar to the previous case, we have

$$
\left(f+g \epsilon_{0}\right)(p \oplus q)=\left(f p+c_{11} g p+c_{12} g q\right) \oplus\left(f q+c_{21} g p+c_{22} g q\right)
$$

and

$$
\left(f+g \epsilon_{1}\right)(p \oplus q)=\left(f p+d_{11} g p+d_{12} g q\right) \oplus\left(f q+d_{21} g p+d_{22} g q\right)
$$

with $c_{11}, c_{12}, c_{21}, c_{22} \in A[x], d_{11}, d_{12}, d_{21}, d_{22} \in A[y]$ such that $c_{11}+c_{22}=0$, $c_{11} c_{22}-c_{12} c_{21}=0, d_{11}(y)=y^{3} c_{11}(1 / y), d_{12}=y^{4} c_{12}(1 / y), d_{21}(y)=y^{2} c_{21}(1 / y)$ and $d_{22}(y)=y^{3} c_{22}(1 / y)$. Hence there are homogeneous polynomials $F_{i j}\left(u_{0}, u_{1}\right)$ for $1 \leq i, j \leq 2$ over $A$ such that $\operatorname{deg}\left(F_{11}\right)=\operatorname{deg}\left(F_{22}\right)=3, \operatorname{deg}\left(F_{12}\right)=4, \operatorname{deg}\left(F_{21}\right)=2$, $c_{i j}(x)=F_{i j}(1, x)$ and $d_{i j}(y)=F_{i j}(y, 1)$.

Since $\mathcal{G}_{s} \cong \mathcal{F}\left(a_{0}: a_{1}: a_{2}\right)$ by assumption, we may assume that $F_{21}\left(u_{0}, u_{1}\right) \equiv$ $a_{0} u_{0}^{2}+a_{1} u_{0} u_{1}+a_{2} u_{1}^{2}\left(\bmod \mathbf{m}_{s}\right)$ and $F_{11}, F_{12}, F_{22} \in \mathbf{m}_{s}\left[u_{0}, u_{1}\right]$. Here $\mathbf{m}_{s}$ denotes the maximal ideal of $\mathcal{O}_{S, s}$.

Since $a_{1}^{2}-4 a_{0} a_{2} \neq 0$, there exists an affine neighborhood $W$ of $s$ such that $F_{21}\left(u_{0}, u_{1}\right)$ factors into two non-proportional linear forms at every point of $W$. Since $F_{22}^{2}$ is divisible by $F_{21}, F_{22}$ is divisible by $F_{21}$ at every point $t \in W$.

For a fixed $t \in W$, let $F_{i j}^{\prime}$ denote the restriction of $F_{i j}$ at $t$. Then $H^{0}\left(U_{0}, \mathcal{G}_{t}^{0}\right)=$ $k[x] e_{0}$ and $H^{0}\left(U_{1}, \mathcal{G}_{t}^{0}\right)=k[y] e_{1}$, where $e_{0}=F_{22}^{\prime}(1, x) / F_{21}^{\prime}(1, x) \oplus(-1)$ and $e_{1}=$ 
$F_{22}^{\prime}(y, 1) / F_{21}^{\prime}(y, 1) \oplus(-1)$. It follows from $e_{0}=e_{1} / y^{2}$ that $\mathcal{G}_{t}^{0} \cong \mathcal{O}(-2)$. Therefore $\mathcal{G}_{t}$ is in $M(\mathcal{O}(-1), \mathcal{O}(-2))$.

Corollary 5.12. Let $C^{\prime}$ be the infinitesimal extension of $\mathbb{P}^{1}$ by $\mathcal{O}(-3)$. Then $\mathfrak{M}(-3)_{\text {ss }}=\mathfrak{M}(-3)_{s}$ has two irreducible components $V_{1}$ and $V_{2}$ such that $V_{1} \cong \mathbb{P}^{2}$ and $\mathfrak{M}(-3) \backslash V_{1}$ is the generalized Jacobian of $C^{\prime}$. The intersection of $V_{1}$ and $V_{2}$ is a conic on $V_{1}$.

For $d>3, \mathfrak{M}(m)_{s s, r e d}$ is the disjoint union of $M\left(\mathcal{O}\left(a_{1}\right), \mathcal{O}\left(b_{1}\right)\right), \ldots$, $M\left(\mathcal{O}\left(a_{r}\right), \mathcal{O}\left(b_{r}\right)\right)$ plus an extra point $\eta$ (class of strictly semistable sheaves) if $m \equiv 0$ $(\bmod 2)$. Here $r=[(d+1) / 2], a_{i}=[(m+1) / 2]+i-1, b_{i}=[m / 2]-i+1$.

Let $X_{i}$ denote the closure of $M\left(\mathcal{O}\left(a_{i}\right), \mathcal{O}\left(b_{i}\right)\right)$ in $\mathfrak{M}(m)_{s s, r e d}$. Then we can deduce the following results by using the same method as before:

1) For every $i, X_{i} \cong \mathbb{P}^{d-1}$.

2) $X_{i} \cap M\left(\mathcal{O}\left(a_{j}\right), \mathcal{O}\left(b_{j}\right)\right)=\emptyset$ for $i<j$.

3) $X_{i} \cap\left(\bigcup_{j=i+1}^{r} X_{j}\right)$ is a quadratic hypersurface of $X_{i}$.

4) If $d+m \equiv 0(\bmod 2)$, then $X_{r}$ is the compactification of the generalized Jacobian $\operatorname{Jac}\left(C^{\prime}\right)$.

ACKnowledgement. This work was inspired by a series of lectures given by Professor Jun Li, who also spent many hours to discuss the subject with the author. We are also grateful to the referee for his or her important comments and suggestions.

\title{
References
}

[1] M.F. Atiyah, Vector bundles over an elliptic curve, Proc. London Math. Soc., 7 (1957), $414-452$.

[2] A. Grothendieck, Sur la classification des fibrés holomorphes sur la sphère de Riemann, Amer. J. Math., 79 (1957), 121-138.

[3] R. Hartshorne, Algebraic geometry, Springer-Verlag, 1977.

[4] D. Huybrechts and M. Lehn, The geometry of moduli spaces of sheaves, Vieweg, 1997.

[5] P.E. Newstead, Lectures on introduction to moduli problems and orbit spaces, Tata Institute of Fundamental Research, Springer-Verlag, 1978.

[6] C.S. Seshadri, Fibrés vectoriels sur les courbes algébriques, Asterisque, 96 (1982), 1-209.

[7] C.T. Simpson, Moduli of representations of the fundamental group of a smooth projective variety I, Publ. Math. Inst. HES, 79 (1994), 47-129.

\author{
INSTITUTE OF MATHEMATICS \\ FUdAN UNIVERSITY \\ ShanghaI, China \\ E-mail: jgyang@srcap.stc.sh.cn
}

